PROCEEDINGS OF THE

AMERICAN MATHEMATICAL SOCIETY

Volume 135, Number 9, September 2007, Pages 2975-2982

S 0002-9939(07)08808-9

Article electronically published on May 9, 2007

\title{
SPECIAL SUBSETS OF THE REALS AND TREE FORCING NOTIONS
}

\author{
MARCIN KYSIAK, ANDRZEJ NOWIK, AND TOMASZ WEISS
}

(Communicated by Julia Knight)

\begin{abstract}
We study relationships between classes of special subsets of the reals (e.g. meager-additive sets, $\gamma$-sets, $C^{\prime \prime}$-sets, $\lambda$-sets) and the ideals related to the forcing notions of Laver, Mathias, Miller and Silver.
\end{abstract}

\section{INTRODUCTION}

This paper is meant to be a sequel of [5] and [11, where the relationships between various classes of small sets and the ideals $\left(l_{0}\right)$ and $\left(m_{0}\right)$ related to the forcing notions of Laver and Miller were studied.

In the present paper, we add the Silver forcing to the list of the forcing notions under consideration. We begin with studying inclusions between the classes of small sets already investigated in [5, and we also consider the $\sigma$-ideal $\left(v_{0}\right)$ related naturally to the forcing notion of Silver. Later we take into consideration more classes of special subsets of the real line and we study their relationships with all the three ideals $\left(l_{0}\right),\left(m_{0}\right)$ and $\left(v_{0}\right)$. We also mention the classical ideal $\left(c r_{0}\right)$ of completely Ramsey-null sets related to the Mathias forcing, solving a problem of J. Brown concerning $\sigma$-sets.

The definitions of Laver and Miller trees as well as the ideals $\left(l_{0}\right),\left(m_{0}\right)$ and the classes of perfectly meager, strongly null and universally null sets are provided in [5]. We continue using all the notation and terminology from above. Here, we shall sometimes abuse terminology by conflating a tree $T$ with the set $[T]$ of its branches.

Let us briefly remind the reader that we treat the Baire space $\omega^{\omega}$ as a cocountable subset of the Cantor space $2^{\omega}$. This identification allows us to think about (sets of branches of) Laver and Miller trees as subsets of $2^{\omega}$. The embedding was described precisely in [5] let us just say that first we identify $\omega^{\omega}$ with the space of strictly increasing sequences of natural numbers $\omega^{\uparrow \omega}$ (and the homeomorphism we use preserves Laver and Miller trees). Then a strictly increasing sequence is identified with the set of its values, which is an element of $[\omega]^{\omega} \subseteq 2^{\omega}$.

Received by the editors March 13, 2006 and, in revised form, June 8, 2006.

2000 Mathematics Subject Classification. Primary 03E05, 03E35, 28E15, 54G99.

Key words and phrases. $\gamma$-set, Rothberger's property, meager-additive set, $\sigma$-set, Laver forcing, Miller forcing, Silver forcing, completely Ramsey-null set.

A part of the research was made when the first author was visiting the Institute of Mathematics of the Polish Academy of Sciences.

The second author was partially supported by grant BW/5100-5-0201-6. 
Sometimes it is more convenient to refer directly to the intermediate step of the construction of that embedding, i.e. to think about (sets of branches of) Laver and Miller trees as subsets of $\omega^{\uparrow \omega}$. We denote by $\mathbb{L}^{\uparrow}$ and $\mathbb{M}^{\uparrow}$ the collections of Laver and Miller trees consisting of strictly increasing sequences.

Although it was said in [5] that the results of that paper hold if one uses any other (but fixed) embedding of $\omega^{\omega}$ onto a co-countable subset of $2^{\omega}$, we do not know whether this remains true for all the theorems proved in the present article.

Definition 1.1. A Silver tree (or a uniform tree) is a perfect tree $T \subseteq 2^{<\omega}$ such that for every $n>0$ either $T \cap 2^{n} \subseteq \operatorname{Split}(T)$ or for all $s, t \in T \cap 2^{n+1}$ we have $s(n)=t(n)$.

The collection of Silver trees will be denoted by $\mathbb{V}$. The family $\mathbb{V}$ ordered by inclusion is known as the Silver forcing. There are two other more popular representations of conditions of this forcing. Observe that each Silver tree corresponds to the set

$$
[f]=\left\{x \in 2^{\omega}: x\lceil\operatorname{dom}(f)=f\}\right.
$$

for a function $f: \operatorname{dom}(f) \rightarrow 2$, where $\operatorname{dom}(f)$ is a co-infinite subset of $\omega$. It also corresponds to the family

$$
\{x \subseteq \omega: a \subseteq x \subseteq b\},
$$

where $a \subseteq b$ are subsets of $\omega$ such that $|b \backslash a|=\omega$. Families of this type are sometimes called doughnuts (see [4]).

Definition 1.2. We shall say that a set $X \subseteq 2^{\omega}$ has the $v_{0}$-property (or $X \in\left(v_{0}\right)$ ) if for every Silver tree $T$, there exists a Silver tree $T^{\prime} \subseteq T$ such that $\left[T^{\prime}\right] \cap X=\emptyset$.

We use standard notation concerning the Mathias forcing and basic Ellentuck sets. For $s \in[\omega]^{<\omega}$ and $A \in[\omega]^{\omega}$ such that $s<A$ (i.e. $\max (s)<\min (A)$ ), we define the basic Ellentuck set $[s, A]$ as follows:

$$
[s, A]=\left\{B \in[\omega]^{\omega}: s \subseteq B \subseteq A\right\} .
$$

Observe that $[s, A]$ is a closed subset of $[\omega]^{\omega}$. When we identify $[\omega]^{\omega}$ with $\omega^{\omega}$ using the identification described in [5, the tree corresponding to $[s, A]$ is in particular a Laver tree (but obviously not every Laver tree is of this type).

Definition 1.3. For a set $X \subseteq 2^{\omega}$, we say that $X$ is completely Ramsey-null, or $X \in\left(c r_{0}\right)$, if for every Ellentuck set $[s, A]$ there exists an Ellentuck set $[t, B] \subseteq[s, A]$, such that $[t, B] \cap X=\emptyset$. Equivalently, for every $[s, A]$, there exists $B \in[A]^{\omega}$ such that $[s, B]$ is disjoint with $X$.

The notion of completely Ramsey-null sets is naturally extended to subsets of $\omega^{\omega}$ using the identification of $\omega^{\omega}$ with $[\omega]^{\omega}$.

\section{Small SETS AND DOUGhnuts}

Theorem 2.1. Every perfectly meager set has the $v_{0}$-property.

Proof. The proof is analogous to the argument from [5] for $\mathcal{P} \mathcal{M} \subseteq\left(m_{0}\right)$ and uses some ideas from 4 .

It was observed in $\left[4\right.$ that every co-meager set in $2^{\omega}$ contains $[T]$ for some $T \in \mathbb{V}$. Notice that if $T \in \mathbb{V}$ and $X \subseteq 2^{\omega}$ is perfectly meager, then $X \cap[T]$ is meager in $[T]$. As $[T]$ is homeomorphic to the space $2^{\omega}$ and the canonical homeomorphism from $2^{\omega}$ to $[T]$ sends Silver trees to Silver trees, we are done. 
Corollary 2.2. Every very meager, strongly meager and additively null set in $2^{\omega}$ has the $v_{0}$-property.

Theorem 2.3. Every universally null set has the $v_{0}$-property.

Proof. We shall use the following lemma.

Lemma 2.4. Every measurable subset of $2^{\omega}$ of positive measure contains a doughnut.

Proof. Let $F \subseteq 2^{\omega}$ be a measurable set of positive measure; without loss of generality we may assume that $F$ is closed. We inductively construct an increasing sequence of natural numbers $\left\langle n_{k}: k \in \omega\right\rangle$ and a sequence $\left\langle s_{k}: k \in \omega\right\rangle$ such that

(1) $s_{k}: \operatorname{dom}\left(s_{k}\right) \rightarrow 2$,

(2) $\operatorname{dom}\left(s_{0}\right)=\left\{0, \ldots, n_{0}-1\right\}$,

(3) $\operatorname{dom}\left(s_{k+1}\right)=\left\{n_{k}+1, \ldots, n_{k+1}-1\right\}$, for $k \in \omega$,

(4) for every $k \in \omega$, there exists $y_{k} \in 2^{\left[n_{k}+1, \infty\right)}$ such that every $x \in\left[\left(\bigcup_{i \leq k} s_{i}\right) \cup\right.$ $\left.y_{k}\right]$ is a density point of $F$.

Observe that if we put $f=\bigcup_{k \in \omega} s_{k}$, then $[f]$ is a doughnut, that is, $f: \operatorname{dom}(f) \rightarrow$ 2 and $\operatorname{dom}(f)$ is a co-infinite subset of $\omega$. Indeed, $\operatorname{dom}(f)=\omega \backslash\left\{n_{k}: k \in \omega\right\}$. Moreover, as $F$ is closed, (4) easily implies that $[f]$ is contained in $F$.

To begin the construction, use the Lebesgue Density Theorem ([12]) to find a density point $y \in F$ and $n_{0} \in \omega$ so large that for $s_{0}=y\left\lceil n_{0}\right.$, we have that

$$
\frac{\lambda\left(\left[s_{0} 0\right] \cap F\right)}{2^{-\left(n_{0}+1\right)}}>\frac{1}{2} \quad \text { and } \quad \frac{\lambda\left(\left[s_{0}^{-1} 1\right] \cap F\right)}{2^{-\left(n_{0}+1\right)}}>\frac{1}{2} .
$$

From these inequalities, it follows easily that the sequences $s_{0}^{\widetilde{0}} 0$ and $s_{0} 1$ have a common extension $y_{0}$ to a density point of $F$.

Suppose now that $n_{i}, s_{i}, y_{i}$ have been constructed for $i \leq k$. As each of $2^{k+1}$ elements of $\left[\left(\bigcup_{i \leq k} s_{i}\right) \cup y_{k}\right]$ is a density point of $F$, we can find $n_{k+1}>n_{k}$ such that putting $s_{k+1}=y_{k} \uparrow\left(\left\{n_{k}+1, \ldots, n_{k+1}-1\right\}\right)$, we get

$$
2^{-\left(n_{k+1}+1\right)} \lambda([s] \cap F)>1-\frac{1}{2^{k+2}}
$$

for every $s \in 2^{\left\{0, \ldots, n_{k}+1\right\}}$ such that $s \in \bigcap_{i=0}^{k+1}\left[s_{i}\right]$. The above inequality implies that all such sequences $s$ have a common extension $y_{k+1}$ to a density point of $F$.

Now, let $X \in \mathcal{U N}$ and let $T \in \mathbb{V}$. Let $\varphi: 2^{\omega} \rightarrow[T]$ be the canonical homeomorphism taking Silver trees to Silver trees. The set $\varphi^{-1}[X]$ has measure zero, so by Lemma 2.4. there exists a Silver tree $\left[T^{\prime}\right]$ disjoint with $\varphi^{-1}[X]$. Then $\varphi\left[\left[T^{\prime}\right]\right]$ is a doughnut contained in $[T]$ and disjoint with $X$.

Corollary 2.5. Every strongly null subset of the Cantor space has the v-property.

\section{Below And ARound strongly null sets}

In [5], we considered the question of whether strongly null sets have $l_{0^{-}}$and $m_{0}$-properties. We proved that the answer is positive in the Baire space with the natural metric, but consistently negative in the Cantor space (with every metric giving this space its standard topology). The question of strongly null sets and the $v_{0}$-property was settled in the previous section.

In this section we consider some stronger properties than being strongly null and the question of whether they imply the $l_{0^{-}}$and/or $m_{0}$-property for subsets of the 
Cantor space. We also consider some other properties of subsets of the Baire space which imply the $l_{0^{-}}$and $m_{0}$-properties.

Proposition 3.1. Every Lusin set (in $2^{\omega}$ and in $\omega^{\omega}$ ) has the $l_{0^{-}}$and the $m_{0^{-}}$ properties.

Proof. We can shrink every Laver (respectively Miller) tree to a meager one. A Lusin set intersects it on a countable subset which can be easily avoided by a yet thinner tree.

Proposition 3.2. Under $C H$ there exists a $C^{\prime \prime}$-set in $2^{\omega}$ which does not have the $l_{0}$-property. Similarly, under $C H$ there exists a $C^{\prime}$-set in $2^{\omega}$ which does not have the $m_{0}$-property.

Proof. In [5], sets concentrated on $\mathbb{Q}$ and not having the $l_{0^{-}}$and $m_{0}$-properties were constructed under $\mathrm{CH}$. If $X$ is concentrated on $\mathbb{Q}$, then $\mathbb{Q} \cup X \in C^{\prime}$.

Every subset of the Baire space with Rothberger's property has the $\left(l_{0}\right)$ - and the $\left(m_{0}\right)$-properties ([5]). Actually, a weaker assumption is sufficient.

Theorem 3.3. If a set $X \subseteq \omega^{\omega}$ has the Menger property, then $X$ has the $l_{0}$-and the $m_{0}$-properties.

Proof. We prove it only for Miller trees; the proof for Laver is analogous.

Let $X \subseteq \omega^{\omega}$ have the Menger property and let $T$ be a Miller tree. Then $X \cap[T]$, as a closed subspace of $X$, has the Menger property too. Let $\left\{s_{k}^{m}: k \in \omega\right\}$ be an injective enumeration of $\operatorname{Split}^{m+1}(T)$. Then $\mathcal{U}_{m}=\left\{\left[s_{k}^{m}\right] \cap X: k \in \omega\right\}$ is a sequence of open coverings of $X \cap[T]$. The Menger property implies 1 that for each $m \in \omega$, there exists $k_{m} \in \omega$ such that $X \cap[T] \subseteq \bigcup\left\{\left[s_{i}^{m}\right]: i<k_{m}\right\}$. Let $T^{\prime}$ be the smallest tree containing all $s_{i}^{m}$, for $i \geq k_{m}$. It is easy to see that $\left[T^{\prime}\right] \cap X=\emptyset$ and $T^{\prime}$ is a Miller subtree of $T$.

A set $X \subseteq 2^{\omega}$ is meager-additive, if $X+M=\{x+y: x \in X, y \in M\}$ is meager, for every meager set $M \subseteq 2^{\omega}$. Every meager-additive set is strongly null, and thus has the $v_{0}$-property.

We give a short proof of the following proposition (compare it with Theorem 24 in [9]).

Proposition 3.4. Every meager-additive set $X \subseteq 2^{\omega}$ is perfectly meager.

Proof. We use the following notation: for an increasing function $f \in \omega^{\omega}$ we shall put $I_{n}^{f}=\{k \in \omega: f(n) \leq k<f(n+1)\}$.

Let $X$ be meager-additive. From Theorem 2.7.17 in 1] we get that for every increasing function $f \in \omega^{\omega}$ there exists $g \in \omega^{\uparrow \omega}$ and $y \in 2^{\omega}$ such that

$$
X \subseteq \bigcup_{m \in \omega} \bigcap_{n>m}\left\{x \in 2^{\omega}: \exists k \in \omega\left(I_{k}^{f} \subseteq I_{n}^{g}\right) \wedge\left(x \uparrow I_{k}^{f}=y \uparrow I_{k}^{f}\right)\right\} .
$$

Let $P \subseteq 2^{\omega}$ be a perfect set and let $T \subseteq 2^{<\omega}$ be a perfect tree such that $P=[T]$. Observe that $\{[s] \cap P: s \in T\}$ is a basis of the relative topology on $P$. Let $f \in \omega^{\omega}$ be an increasing function such that for every $t \in T \cap 2^{f(k)}$ there exists at least two different elements $s_{1}, s_{2} \in T \cap 2^{f(k+1)}$ extending $t$.

\footnotetext{
${ }^{1}$ Some authors require that open covers considered in the Menger property do not have a finite subcover. Observe that if one of our covers has a finite subcover, we are also done.
} 
To show that $X \cap P$ is meager in $P$, it is enough to show that for every $m \in \omega$ the set

$$
F_{m}=P \cap \bigcap_{n>m}\left\{x \in 2^{\omega}: \exists k \in \omega\left(I_{k}^{f} \subseteq I_{n}^{g}\right) \wedge\left(x \uparrow I_{k}^{f}=y \uparrow I_{k}^{f}\right)\right\}
$$

is nowhere dense in $P$. To this end, let $t \in T$. By extending $t$ we may always assume that $|t|=f(k)$, where $k$ is minimal such that $I_{k}^{f} \subseteq I_{n}^{g}$, for some $n>m$. Let $j \in \omega$ be maximal such that $I_{k+j}^{f} \subseteq I_{n}^{g}$. We can find an extension $t_{0} \supseteq t$ such that $t_{0} \in T$, $\left|t_{0}\right|=f(k+1)$ and $t_{0}\left\lceil I_{k}^{f} \neq y \uparrow I_{k}^{f}\right.$. This is possible since by the choice of $f$ we have two candidates for such an extension, so one of them is different from $y \uparrow I_{k}^{f}$. Then we apply the same procedure to find $t_{i}$ for $i \leq j$, such that $t_{i+1}$ extends $t_{i}$, $\left|t_{i}\right|=f(k+i+1)$ and $t_{i}\left\lceil I_{k+i}^{f} \neq y\left\lceil I_{k+i}^{f}\right.\right.$. It is clear that $\left[t_{j}\right] \cap F_{m}=\emptyset$.

Corollary 3.5. Every meager-additive set in $2^{\omega}$ has the $m_{0}$-property.

Theorem 3.6. Under $C H$ there exists a meager-additive set in $2^{\omega}$ which does not have the $l_{0}$-property.

Proof. We use similar arguments to those from the proof of Theorem 4.3 in [10. We need the following lemma.

Lemma 3.7. For every $M \in \mathcal{M}$, there exists $M^{\prime} \in \mathcal{M}$ such that $M+\left(2^{\omega} \backslash M^{\prime}\right) \in$ $\left(l_{0}\right)$.

Proof. Let $M \subseteq 2^{\omega}$ be meager. By considering a translate of $M$ (which can always be done), we can assume that there exists a partition of $\omega$ into consecutive finite intervals $\left\{I_{k}: k \in \omega\right\}$ such that $M \subseteq\left\{x \in 2^{\omega}: \forall^{\infty} k \in \omega x \uparrow I_{k} \not \equiv 0\right\}$ (see 11). Let $J_{k}=I_{2 k} \cup I_{2 k+1}$ and define $M^{\prime}=\left\{x \in 2^{\omega}: \forall^{\infty} k \in \omega x \uparrow J_{k} \not \equiv 0\right\}$. Clearly, $M^{\prime} \in$ $\mathcal{M}$.

To check that $M+\left(2^{\omega} \backslash M^{\prime}\right) \in\left(l_{0}\right)$, assume that $T \in \mathbb{L}^{\uparrow}$. We define $T^{\prime} \subseteq T$ by induction on the length of its elements. Let $\operatorname{stem}\left(T^{\prime}\right)=\operatorname{stem}(T)$. For $t \supseteq \operatorname{stem}\left(T^{\prime}\right)$ already included in $T^{\prime}$, put in $T^{\prime}$ the elements of the set

$$
\left\{n \in \omega:\left(t^{\frown} n \in T\right) \wedge\left(n \geq \min \left(J_{k+1}\right)\right)\right\},
$$

where $J_{k}$ contains the last term of $t$. Observe that for every $x \in\left[T^{\prime}\right]$ (treated as a subset of $\omega$ ) and for almost all $k \in \omega$, we have $\left|x \cap J_{k}\right| \leq 1$.

We will show that $\left[T^{\prime}\right] \cap\left(M+\left(2^{\omega} \backslash M^{\prime}\right)\right)=\emptyset$. Let $x \in\left[T^{\prime}\right]$ and assume towards a contradiction that $x=m+m^{\prime}$, where $m \in M$ and $m^{\prime} \notin M^{\prime}$. By definition of $M$ and $M^{\prime}$, there exists an arbitrarily large $k \in \omega$ such that $m\left\lceil I_{2 k} \not \equiv 0\right.$ and $m\left\lceil I_{2 k+1} \not \equiv 0\right.$, but $m^{\prime}\left\lceil J_{k} \equiv 0\right.$. Then $x \uparrow J_{k}=\left(m+m^{\prime}\right) \uparrow J_{k}=m \uparrow J_{k}$ must take value 1 at least two times, which contradicts the fact that $x \in\left[T^{\prime}\right]$.

The rest of the proof is analogous to the argument from [10], but we present it here for completeness. Assuming $\mathrm{CH}$, let $\left\{M_{\alpha}: \alpha \in \omega_{1}\right\}$ be an enumeration of all meager $F_{\sigma}$ sets and let $\left\{T_{\alpha}: \alpha \in \omega_{1}\right\}$ be an enumeration of all Laver trees. For every $M_{\alpha}$ find $M_{\alpha}^{\prime}$ such that $M_{\alpha}+\left(2^{\omega} \backslash M_{\alpha}^{\prime}\right) \in\left(l_{0}\right)$. In step $\alpha<\omega_{1}$, pick $x_{\alpha} \in\left[T_{\alpha}\right] \backslash \bigcup_{\xi<\alpha}\left(M_{\xi}+\left(2^{\omega} \backslash M_{\xi}^{\prime}\right)\right)$. If we put $X=\left\{x_{\alpha}: \alpha \in \omega_{1}\right\}$, then obviously $X \notin\left(l_{0}\right)$. To see that $X$ is meager additive, observe that $X+M_{\alpha} \subseteq\left(\left\{x_{\xi}: \xi \leq \alpha\right\}+\right.$ $\left.M_{\alpha}\right) \cup M_{\alpha}^{\prime}$.

If $X$ is a $\gamma$-set and $X \subseteq \omega^{\omega}$, then $X$ has Rothberger's property, so $X$ has the $l_{0^{-}}$ and the $m_{0}$-properties. However if $X \subseteq 2^{\omega}$ is a $\gamma$-set, then $X \cap[\omega]^{\omega}$ need not be 
a $\gamma$-set, so it is not obvious that $\gamma$-sets in $2^{\omega}$ have these properties. Rothberger's property can be destroyed by subtracting the countable set $[\omega]^{<\omega}$, and in [5] we have seen that this affects the $l_{0^{-}}$and $m_{0}$-properties. Nevertheless, we can still prove that $\gamma$-sets in the Cantor space have these properties.

Theorem 3.8. Every $\gamma$-set in $2^{\omega}$ has the $l_{0}$-and the $m_{0}$-properties.

Proof. We shall use the following lemma.

Lemma 3.9 (see Theorem 2.1 in [10]). Let $X \subseteq 2^{\omega}$ be a $\gamma$-set. There exists $A \in[\omega]^{\omega}$ such that $[A]^{\omega} \cap X=\emptyset$. In particular, there exists $T \in \mathbb{L}$ such that $[T] \cap X=\emptyset$.

Lemma 3.10. For every $T \in \mathbb{L}^{\uparrow}$, there exists a homeomorphism $\psi: 2^{\omega} \rightarrow \overline{[T]} \subseteq 2^{\omega}$ such that for every Laver tree $T^{\prime}$, the set $\psi\left[\left[T^{\prime}\right]\right] \subseteq[\omega]^{\omega}$ and is the set of branches of a Laver tree.

Similarly, for every $T \in \mathbb{M}^{\uparrow}$, there exists a homeomorphism $\psi: 2^{\omega} \rightarrow \overline{[T]} \subseteq 2^{\omega}$ such that for every Miller tree $T^{\prime}$, the set $\psi\left[\left[T^{\prime}\right]\right] \subseteq[\omega]^{\omega}$ and is the set of branches of a Miller tree.

Proof. We prove only the first part; the other one is similar.

Recall that we embed the set $[T]$ (for a Laver tree $T$ consisting of strictly increasing functions) into $[\omega]^{\omega} \subseteq 2^{\omega}$, naturally identifying a function with its range. The same can be done with elements of such a tree $T$ (i.e. finite increasing sequences of natural numbers), thus we may think of $T$ as a subset of $\mathbb{Q}=\left\{x \in 2^{\omega}: \forall^{\infty} n \in \omega x(n)=0\right\} \subseteq 2^{\omega}$. Under this identification we have $T \cup$ $[T]=\overline{[T]} \subseteq 2^{\omega}$.

Now we define $\widehat{\psi}: S \rightarrow T$, where $S \subseteq 2^{<\omega}$ consists of finite binary sequences ending with 1 and finite sequences constantly equal to 0 by induction on the number of ones in $t \in S$. If $t$ is constantly equal to 0 , we put $\widehat{\psi}(t)=\operatorname{stem}(T)$. Assume that $t \in S, m=|t|-1, t(n-1)=1, t(m)=1$ and $t(i)=0$ for $n-1<i<m$. As $\widehat{\psi}(t\lceil n) \in T$ has been already defined, we put $\widehat{\psi}(t)=\widehat{\psi}(t\lceil n) \frown k$, where $k$ is the $m-n-1$-th element of the set $\{j \in \omega: \widehat{\psi}(t\lceil n) \frown j \in T\}$. This is a correct definition, since $T \in \mathbb{L}$.

Observe that if $s, t \in S$ and $s \subseteq t$, then $\widehat{\psi}(s) \subseteq \widehat{\psi}(t)$. Thus, it makes sense to define a function $\psi: 2^{\omega} \rightarrow[T] \cup T$ as follows:

$$
\psi(x)=\bigcup\{\widehat{\psi}(x\lceil n): n \in \omega \wedge x(n-1)=1\} .
$$

This function is easily seen to be one-to-one and continuous. It also takes Laver trees to Laver trees as it preserves infinite-branching nodes.

Now let $X \subseteq 2^{\omega}$ be a $\gamma$-set and let $T$ be a Laver tree. Without loss of generality we may assume that $T \in \mathbb{L}^{\uparrow}$. Then $X \cap \overline{[T]}$ is a $\gamma$-set. Using $\psi$ from Lemma 3.10 , we obtain that $\psi^{-1}[X \cap \overline{[T]}]$ is a $\gamma$-set. Then from Lemma 3.9 we get a Laver tree $T^{\prime}$ disjoint with $\psi^{-1}[X \cap \overline{[T]}]$ and consequently $\psi\left[\left[T^{\prime}\right]\right]$ is a Laver tree disjoint with $X$.

The second part (i.e. for Miller trees) is proved in a similar way.

Remark 3.11. Notice that we essentially proved that if $P$ is a class of subspaces of $2^{\omega}$ closed under taking closed subsets and homeomorphisms, then

$$
P \subseteq\left(c r_{0}\right) \Longrightarrow P \subseteq\left(l_{0}\right) \Longrightarrow P \subseteq\left(m_{0}\right) \Longrightarrow P \subseteq\left(s_{0}\right) .
$$


An analogous argument shows that for such a class, we also have that

$$
P \subseteq\left(v_{0}\right) \Longrightarrow P \subseteq\left(s_{0}\right) .
$$

A similar observation concerning the algebras related to these notions of forcing was made in [2].

Remark 3.12. By a modification of the proof of Theorem 2.2 from [10] one can show that if $X$ is a $T^{\prime}$-set and $Y$ is strongly meager (see [10] for definitions), then $X+Y$ has the $\left(l_{0}\right)-$ and the $\left(m_{0}\right)$-properties. This obviously gives a different argument for the fact proved in Theorem 3.8. We plan to treat the subject of inclusions between algebraic sums of special sets and forcing ideals in a separate paper.

\section{Special Sets Related to Borel hierarchy}

In this section we study special sets defined in terms of Borel hierarchy. A set $X$ is said to be a $Q$-set, if every subset of $X$ is a relative $G_{\delta}, X$ is a $\sigma$-set if every relative $F_{\sigma}$ in $X$ is a relative $G_{\delta}$, and it is a $\lambda$-set if every countable subset of $X$ is a relative $G_{\delta}$ in $X$. We shall say that a set $X$ is a $\lambda^{\prime}$ set, if $X \cup Y$ is a $\lambda$-set for every countable $Y$. Properties $Q, \sigma$ and $\lambda$ are topological; $\lambda^{\prime}$ makes sense for subsets of a given topological space, which should always be clear from the context.

Inclusions between these classes and the ideal $\left(c r_{0}\right)$ were investigated by Brown in 3 .

Proposition 4.1. Every $Q$-set has the $l_{0^{-}}, m_{0^{-}}$and $v_{0}$-properties.

Proof. If $X$ is a $Q$-set, then $|X|<\mathfrak{c}$.

Proposition 4.2. Every $\lambda$-set (in $\omega^{\omega}$ and in $2^{\omega}$ ) has the $m_{0}$ - and the $v_{0}$-properties. In particular, every $\sigma$-set has these properties.

Proof. Every $\lambda$-set is perfectly meager (see [6]).

As the relations of $\lambda$ - and $\sigma$-sets with $\left(m_{0}\right)$ and $\left(v_{0}\right)$ have been clarified, we are going to consider relations of these classes with $\left(l_{0}\right)$ and $\left(c r_{0}\right)$.

The next theorem answers in particular a question of J. Brown from [3] about $\sigma$-sets and the $\left(c r_{0}\right)$ ideal. The other part, concerning the $\left(l_{0}\right)$ ideal, was proved independently by A. W. Miller in [8].

Theorem 4.3. Every $\sigma$-set (in $2^{\omega}$ and in $\omega^{\omega}$ ) has the $\left(l_{0}\right)$ and the $\left(c r_{0}\right)$-properties.

Proof. First observe that we may consider $\sigma$-sets contained in $\omega^{\omega}$. For $\left(l_{0}\right)$ and $\left(c r_{0}\right)$, only the part contained in $[\omega]^{\omega}$ is relevant and every subset of a $\sigma$-set is a $\sigma$-set.

Next, notice that the inclusion $\sigma \subseteq\left(c r_{0}\right)$ is equivalent to the statement: for every $\sigma$-set $X$, there exists an Ellentuck set disjoint with $X$. This follows from the fact that an Ellentuck set is a closed set homeomorphic to the whole space with the canonical homeomorphism sending Ellentuck sets to Ellentuck sets. Therefore to prove the theorem it is enough to show that for every $\sigma$-set there exists a disjoint Ellentuck set.

So let $X \subseteq[\omega]^{\omega}$ be a $\sigma$-set and let $\left\{t_{n}: n \in \omega\right\}$ be a fixed bijective enumeration of $2^{<\omega}$. Consider $D=\left\{A \in[\omega]^{\omega}: \forall n, m \in A t_{n} \subseteq t_{m} \vee t_{m} \subseteq t_{n}\right\}$. Let $f: D \rightarrow 2^{\omega}$ be a function defined by the formula

$$
f(A)=\bigcup\left\{t_{n}: n \in A\right\} .
$$


Clearly, $f$ is continuous, so by a result of Recław (see [7), it cannot map $D \cap X$ onto $2^{\omega}$. So let $y \in 2^{\omega}$ be such that for no $A \in D \cap X, f(A)=y$. If we put $B=\left\{n \in \omega: t_{n} \subseteq y\right\}$, then $[B]^{\omega}=[\emptyset, B] \subseteq D$ and $[\emptyset, B] \cap X=\emptyset$.

The inclusion $\sigma \subseteq\left(l_{0}\right)$ follows from Remark 3.11 .

Proposition 4.4. Under $C H$ there exists a $\lambda^{\prime}$-set in $\omega^{\omega}$ which does not have the $l_{0}$-property. In particular, this set is a $\lambda$-set in $2^{\omega}$.

Proof. It follows from the fact that an $\omega_{1}$-scale (i.e. strictly increasing in the sense of $\leq^{*}$ and dominating $\omega_{1}$-sequence of elements of $\omega^{\omega}$ ) is a $\lambda^{\prime}$-set in $\omega^{\omega}$ (see [6]). An $\omega_{1}$-scale not having the $l_{0}$-property was constructed under $\mathrm{CH}$ in [5].

Proposition 4.5. Every $\lambda^{\prime}$-set in $2^{\omega}$ has the $l_{0}$-property.

Proof. This is a modification of Brown's argument from [3]. If $X \subseteq 2^{\omega}$ is a $\lambda^{\prime}$-set, then $X \cup \mathbb{Q}$ is a $\lambda$-set. Consequently $\mathbb{Q}$ is a $G_{\delta}$ set in $X \cup \mathbb{Q}$, so $X \cap \omega^{\omega}$ is a bounded set in $\omega^{\omega}$. It is easy to see that each bounded set has the $l_{0}$-property.

\section{REFERENCES}

[1] T. Bartoszyński and H. Judah, Set Theory: On the Structure of the Real Line, A K Peters, Ltd, Wellesley, Massachusetts, 1995. MR.1350295 (96k:03002)

[2] J. Brendle and B. Löwe, Solovay-type characterizations for forcing-algebras, J. Symbolic Logic 64 (1999), no. 3, 1307-1323. MR 1779764 (2001h:03086)

[3] J. B. Brown, The Ramsey sets and related sigma algebras and ideals, Fundamenta Mathematicae 136 (1990), no. 3, 179-185. MR1095690(92a:04003)

[4] L. Halbeisen, Making doughnuts of Cohen reals, Mathematical Logic Quarterly 49 (2003), no. 2, 173-178. MR.1961459(2004b:03080)

[5] M. Kysiak and T. Weiss, Small subsets of the reals and tree forcing notions, Proceedings of the American Mathematical Society 132 (2004), no. 1, 251-259. MR2021269 (2004j:03062)

[6] A. W. Miller, Handbook of set-theoretic topology, ch. Special Subsets of the Real Line, pp. 201233, North-Holland, Amsterdam, 1984.

[7] _ Special sets of reals, Israel Mathematical Conference Proceedings, vol. 6, 1993. MR:1234286 (95a:03064)

[8] $\longrightarrow$ A hodgepodge of sets of reals, Preprint (2006).

[9] A. Nowik, M. Scheepers, and T. Weiss, The algebraic sums of sets of real numbers with strong measure zero sets, Journal of Symbolic Logic 63 (1998), no. 1, 301-324. MR.1610427 (99c:54049)

[10] A. Nowik and T. Weiss, On the Ramseyan properties of some special subsets of $2^{\omega}$ and their algebraic sums, The Journal of Symbolic Logic 67 (2002), no. 2, 547-556. MR.1905154 (2003c:03087)

[11] Strongly meager sets of the real numbers and tree forcing notions, Proceedings of the American Mathematical Society 130 (2002), no. 4, 1183-1187. MR1873795 (2002j:03049)

[12] J. C. Oxtoby, Measure and category, Graduate Texts in Mathematics, vol. 2, Springer-Verlag, New York - Berlin, 1980. MR0584443 (81j:28003)

Institute of Mathematics, Warsaw University, ul. Banacha 2, 02-097 Warszawa, POLAND

E-mail address: mkysiak@mimuw.edu.pl

Institute of Mathematics, University of Gdańsk, Wita Stwosza 57, 80-952 Gdańsk, POLAND

E-mail address: nowik@math.uni.gda.pl

Institute of Mathematics and Physics, Akademia Podlaska, ul. 3-go Maja, 08-110 Siedlce, Poland

E-mail address: tomaszweiss@o2.pl 